\title{
A Novel Method for Chronic Social Defeat Stress in Female Mice
}

\author{
Alexander Z Harris*,1,2, Piray Atsak 1,2,3,4,5, Zachary H Bretton ${ }^{1,5}$, Emma S Holt', Raisa Alam', \\ Mitchell P Morton ${ }^{1,2}$, Atheir I Abbas ${ }^{1,2}$, E David Leonardo', , Scott S Bolkan', René Hen ${ }^{1,2}$ and \\ Joshua A Gordon ${ }^{1,2,6}$ \\ 'Department of Psychiatry, College of Physicians and Surgeons, Columbia University, New York, New York, USA; ${ }^{2}$ Division of Integrative \\ Neuroscience, New York State Psychiatric Institute, New York, New York, USA; ${ }^{3}$ Department of Cognitive Neuroscience, Radboud University \\ Medical Center, Nijmegen, The Netherlands; ${ }^{4}$ Donders Institute for Brain, Cognition and Behaviour, Radboud University, Nijmegen, The \\ Netherlands
}

Historically, preclinical stress studies have often omitted female subjects, despite evidence that women have higher rates of anxiety and depression. In rodents, many stress susceptibility and resilience studies have focused on males as one commonly used paradigm-chronic social defeat stress - has proven challenging to implement in females. We report a new version of the social defeat paradigm that works in female mice. By applying male odorants to females to increase resident male aggressive behavior, we find that female mice undergo repeated social defeat stress and develop social avoidance, decreased sucrose preference, and decreased time in the open arms of the elevated plus maze relative to control mice. Moreover, a subset of the female mice in this paradigm display resilience, maintaining control levels of social exploration and sucrose preference. This method produces comparable results to those obtained in male mice and will greatly facilitate studying female stress susceptibility.

Neuropsychopharmacology (2018) 43, 1276-1283; doi:I0.1038/npp.2017.259; published online 6 December 2017

\section{INTRODUCTION}

Although women have higher rates of stress-associated disorders, including anxiety and depression (Kessler et al, 1994; Kessler et al, 2003), the majority of preclinical research investigating the effects of stress has excluded female subjects (Beery and Zucker, 2011; Lebron-Milad and Milad, 2012). Although several studies have reported sex differences in the neural and behavioral responses to stress (Autry et al, 2009; Gruene et al, 2015; Hodes et al, 2015; Shansky et al, 2010), many of the models developed to study the effects of stress in rodents optimally work in males (Haller, 1999; Kokras and Dalla, 2014; Shansky, 2015; Trainor, 2011). Among chronic stress paradigms, chronic social defeat stress (CSDS) has been widely used to investigate the neural mechanisms underlying anxiety and depression-like behaviors (Russo and Nestler, 2013). In this model, experimental animals repeatedly undergo social defeat for several days (Kudryavtseva et al, 1991). In male mice, this experience reliably produces

*Correspondence: Dr AZ Harris, Department of Psychiatry, College of Physicians and Surgeons, Columbia University, 105I Riverside Drive, Unit 87, Kolb Annex Room 140, New York, NY 10032, USA. Tel: +6467747I I I, E-mail: ah2835@cumc.columbia.edu

${ }^{5}$ These authors contributed equally to this work.

${ }^{6}$ Current address: National Institute of Mental Health, Bethesda, MD 20892, USA.

Received 31 July 2017; revised 18 October 2017; accepted 24 October 2017; accepted article preview online I November 2017 groups of susceptible mice with reduced social investigation and sucrose preference as well as groups of resilient mice that pursue these behaviors at control levels (Berton et al, 2006; Krishnan et al, 2007). In males, susceptible and resilient mice differ at the molecular, cellular, brain metabolite, and circuit level (Chaudhury et al, 2013; Krishnan et al, 2007; Larrieu et al, 2017; Wook Koo et al, 2016). However, it remains unclear whether these findings extend to female mice. To date, this model has proven difficult to implement in typical female mice since under most conditions neither male nor female resident mice attack intruder females. Thus, past efforts to apply social defeat stress in female rodents have either relied on logistically difficult methods (Bourke and Neigh, 2012; Haller, 1999) or non-standard species (Trainor et al, 2011), limiting the ability to incorporate females into studies of stress-susceptibility.

To address this issue, we have developed an accessible model of social defeat for use in female mice. To do so, we exploited the known propensity of odorants and pheromones in urine to increase male mouse aggressive behavior (Chamero et al, 2007; Mugford and Nowell, 1970). Specifically, we applied male urine to females to induce aggressive male CD1 mice to repeatedly attack female intruder C57BL/6J mice over the course of 10 days. This method closely follows a standard protocol used for CSDS in male mice (Golden et al, 2011). We anticipate that the development of this easy-to-use method for social defeat stress in female mice will greatly facilitate the inclusion of female subjects in stress research. 


\section{MATERIALS AND METHODS}

\section{Animals}

A total of 64 female C57BL/6J adult mice aged 3 to 4 months (Jackson laboratories) were used as subjects, 39 females in the experimentally stressed group, and 25 females in the control group. Twenty-two CD1 retired breeder adult males (Charles River laboratories) were used as aggressors and social interaction (SI) probands. CD1 males were prescreened over the course of 3 days to ensure that they reliably attacked male mice with a latency of under $1 \mathrm{~min}$. Except for the 10-day aggression period and the subsequent SI, C57BL/6J mice were cohoused in groups of 5 from the time that they were received (aged 10 weeks) and CD1 mice were housed alone. Stressed mice were regrouped with the same cagemates after undergoing CSDS. All mice were maintained on a 12-hour light/dark cycle and given ad libitum food and water unless otherwise noted. All behavioral testing was done during the light cycle. All procedures were done in accordance with guidelines approved by the Institutional Animal Care and Use Committees at Columbia University and the New York State Psychiatric Institute.

\section{Urine Collection}

CD1 mice were placed in plastic containers with raised wire caging on the bottom for one hour. Urine excreted during this time was collected from the bottom of the container and stored at $4{ }^{\circ} \mathrm{C}$.

\section{Behavioral Measurements}

Social defeat. In the classical CSDS paradigm, mice undergo 10 days of 5 to 10 -min exposure to a different resident aggressive mouse each day followed by $24 \mathrm{~h}$ of cohousing with the aggressor across a perforated, transparent, divider. To adapt this paradigm for female mice, we treated each experimental female mouse with the urine of a male CD1 mouse unknown to the resident $\mathrm{CD} 1$ aggressors. Each female mouse was paired with the urine of a particular CD1 mouse throughout the entire course of social defeat. Urine was applied at the base of the tail $(20 \mu \mathrm{l})$ and on the vaginal orifice $(20 \mu \mathrm{l})$ and immediately placed in a resident aggressive CD1 mouse's homecage for a 5-min interaction session. Throughout this session, the mice were monitored for aggressive interactions and mounting behavior. Aggressive bouts were defined as beginning with the resident CD1 attacking the intruder mouse and ending when the female mouse made an escape leap. The session was terminated immediately if mounting behavior occurred, or after $5 \mathrm{~min}$ of interaction if no mounting behavior occurred. Mounting occurred in $11 \%$ of interactions at an average latency of $3 \mathrm{~min} 10 \mathrm{~s}$ (range 1:23-5:00 min). In no circumstances did mounting lead to pregnancy. After each session, female mice were housed for $24 \mathrm{~h}$ across a perforated, transparent divider from the male aggressor. This process of exposure and cohousing was repeated daily for 10 days. Control mice were cohoused across a divider with a different aggressive mouse for 10 days, but had no physical contact with the aggressive mice. Nineteen of the twenty-five control mice also had male urine applied to them. We conducted the social defeat paradigm in three separate cohorts.

Social interaction. Mice were tested for social affiliation after social defeat using a social interaction test (Golden $e t$ al, 2011) on day 11. To see the persistence of CSDS effects on social interaction, we also repeated the social interaction test 40 days after defeat in 17 mice. In the social interaction test, mice explored a chamber $(40 \mathrm{~cm} \times 40.5 \mathrm{~cm})$ containing an empty enclosure (10 $\mathrm{cm}$ diameter steel pencil cup) for $2.5 \mathrm{~min}$, followed by exploration of the same chamber for an additional $2.5 \mathrm{~min}$ after the introduction of a novel mouse (CD1 male) into the enclosure. An interaction zone was defined by a $30 \mathrm{~cm} \times 14 \mathrm{~cm}$ area around the enclosure. The SI ratio was calculated by dividing the time spent in the interaction zone with the novel mouse present by the time in the interaction zone with the empty enclosure.

Sucrose preference. Mice were habituated to a $1 \%$ sucrose solution in their home cages for 4 days. During sucrose preference testing, the mice were fluid restricted for $8 \mathrm{~h}$ daily while socially housed. They were then placed in individual cages containing two bottles daily for the remaining $16 \mathrm{~h}$. On day 1 both bottles contained water and on the subsequent 2 days, the bottles contained either water or a $1 \%$ sucrose solution and were counterbalanced for position. Each morning the bottles were weighed and sucrose preference over the course of the 2 days was calculated as the percentage of sucrose solution out of total fluid consumed $(100 \times$ sucrose/[sucrose+water]).

Elevated plus maze. A subset of mice $(n=35)$ was tested in the elevated plus maze (EPM), which was $31 \mathrm{~cm}$ above the floor with two closed arms $(7.6 \mathrm{~cm}$ wide $\times 28 \mathrm{~cm}$ long, $15 \mathrm{~cm}$ high walls) and two open arms $(7.6 \mathrm{~cm}$ wide $\times 28 \mathrm{~cm}$ long, $1 \mathrm{~cm}$ high lip). Mice explored the EPM for $5 \mathrm{~min}$ in 300 lux lighting. To derive the combined behavioral index (CBI) we used the formula $\mathrm{CBI}=(z$-scored $(\mathrm{SI})+z$-scored (sucrose preference) $+z$-scored $(\mathrm{EPM})) / 3$, with $z$-score for each behavior calculated as $z$-scored $(X)=\left(\mathrm{X}_{i}\right.$-mean $\left.(X)\right) /$ standard deviation $(X)$, where $X=$ the SI ratio, sucrose preference or EPM open arm exploration.

\section{Corticosterone Measurement and Estrous Cycle Phase Determination}

Blood collection and corticosterone measurement. Thirty minutes after the first day of social defeat, blood was collected from the sub-mandibular vein into tubes containing 0.5M EDTA. We collected blood from the control mice and stressed mice on the same day and matched the time of day by interleaving control and stressed mice. Samples were placed on ice until centrifugation $\left(3000 \mathrm{rpm}\right.$ for $15 \mathrm{~min}$ at $4^{\circ}$ C) and the supernatant was stored at $-80^{\circ} \mathrm{C}$. Plasma corticosterone levels were measured using an ELISA kit (Enzo Life Sciences, Farmingdale, NY).

Estrous cycle. To determine the phase of the estrous cycle during daily social defeat, we used the visual inspection method (Byers et al, 2012). On SI days, the phase of the estrous cycle was determined histologically using wet smears 
obtained from vaginal lavage (Caligioni, 2009). We compared the two methods and determined that the visual inspection method reliably distinguishes between pro-estrus/ estrus and metestrus/diestrus, but does not accurately discriminate pro-estrus from estrus nor metestrus from diestrus (data not shown). As a result, we report grouped pro-estrus/estrus data and grouped metestrus/diestrus data.

\section{Analysis}

Unless otherwise noted, all behavior tests and aggression trials were recorded in 100 lux lighting using Neuralynx Cheetah (Bozeman, MT) for video tracking. Mouse tracking data was extracted from video files using idTracker (PerezEscudero et al, 2014) and imported to Matlab for further analysis using custom scripts. Data across all conditions were grouped and outliers removed using the Tukey method. Means were compared using non-parametric tests (Wilcoxon-rank sum test or Kruskal-Wallis test, with post hoc Wilcoxon-rank sum test and Bonferroni correction).

\section{RESULTS}

Female mice were subjected to 10 days of social defeat, followed by successive daily behavioral testing in the social interaction and sucrose preference tests, as well as the elevated plus maze for a subset of the animals (Figure 1a). Introducing female mice paired with aggressive male scent induced attacks from resident male mice to the female mice (Supplementary Video 1) and subordinate postures from female mice (Supplementary Video 2). Approximately half of resident-intruder pairings resulted in aggression, whereas courtship behavior (assayed by mounting) occurred infrequently (Figure 1b). This distribution of aggressive and mounting behavior did not vary across phases of the estrous cycle (Figure 1b; $n=220$ interactions, $\chi^{2}=0.2341, P>0.89$ ). As expected for a stressful experience, female mice encountering the aggressive males showed elevated corticosterone levels relative to control mice, measured on the first day of social defeat (Figure 1c; $n=25,38$; Wilcoxon-rank sum, $P=0.0043$ ), and this social aggression-induced increase persisted to day 10 (Supplementary Figure 1; $n=25,38$, Wilcoxon-rank sum, $P=0.029)$. Over the course of 10 days, resident male mice attacked female mice a median of 5 days (range 1-10; Figure 1d). Aggressive days consisted of a median of 2 aggressive bouts/session (range 1-9 bouts). The median duration of an aggressive bout was $5.3 \mathrm{~s} \quad(n=50$ analyzed bouts; range $2-9.7 \mathrm{~s}$ ). We further characterized the interactions between resident male and intruder female mice, drawing on the classic literature describing aggression and courtship behavior (Supplementary Table 1) (McGill, 1962; Miczek and O'Donnell, 1978).

Previous studies using social defeat stress in male mice have reported that stress-induced effects on behavior only emerge after multiple days of aggression (Chaudhury et al, 2013; Wook Koo et al, 2016). To determine the threshold of aggression needed to induce stress effects, we calculated a CBI by averaging the $z$-scores of sucrose preference, SI and EPM open arm exploration. We then grouped the mice by the number of aggression days they experienced and compared the CBI with control mice, revealing that 4 days or more of aggression induced a significant reduction in CBI (Supplementary Figure 2). As a result, we excluded mice that experienced fewer than 4 days of aggression $(n=9 / 39)$ from subsequent analyses.

Female mice that underwent repeated defeat selectively decreased their exploration of a novel mouse relative to control mice, despite intact exploration of a non-social target (Figure 2a; Kruskal-Wallis, $\chi^{2}=15.3 ; P=0.0015$ for main effect of group, post hoc Wilcoxon-rank sum $P=0.001$ for exploration of mouse; $P=0.51$ for exploration of a non-social target; $(n=25,30)$, Bonferroni corrected critical value $=$ $0.0125)$. As a result, the stressed mice had reduced SI ratios (Golden et al, 2011) measured 1 day post defeat (see

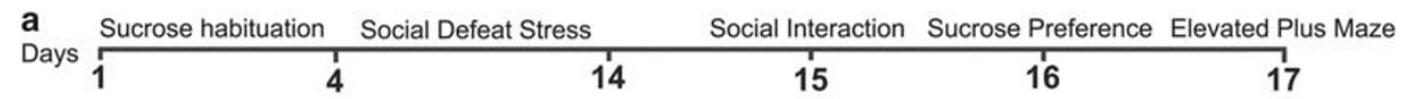

b

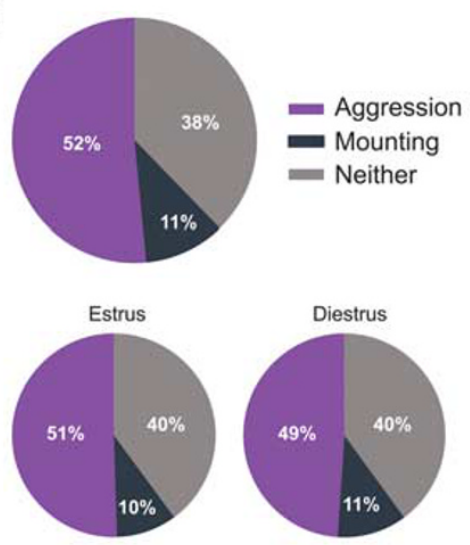

C

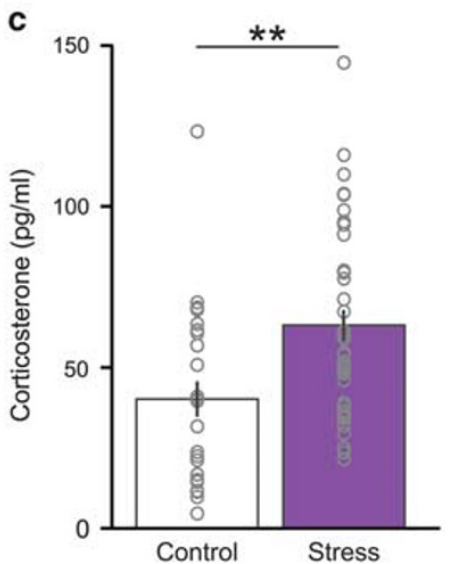

d

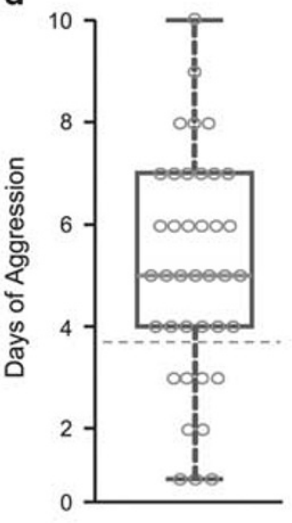

Figure I Resident male mice attack female intruders paired with male scent. (a) Experimental timeline. (b) Rates of aggression and mounting during resident-intruder sessions ( $n=39$ mice, 10 sessions per mouse) for all sessions (upper charts), and split by estrous phase (lower charts). (c) Day I plasma corticosterone responses to aggression (Wilcoxon-rank sum, ${ }^{*} * \mathrm{P}<0.0 \mathrm{I} ; n=25$ and 39 for control and stressed animals, respectively). Error bars represent SEM. (d) Number of aggressive interactions over 10 days of social defeat, for each animal $(n=39)$. Error bars represent I.5 $\times$ the interquartile range (IQR). 
a

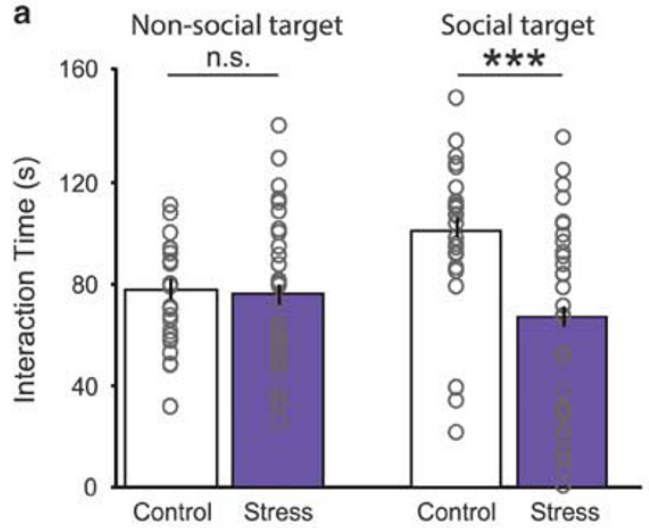

b

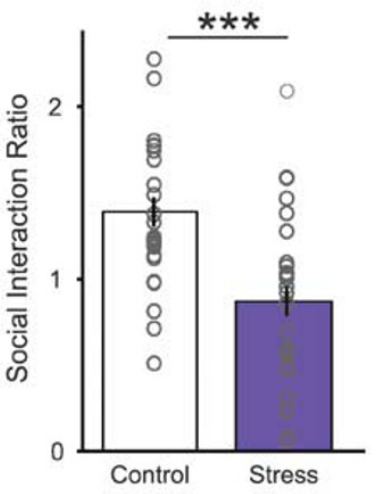

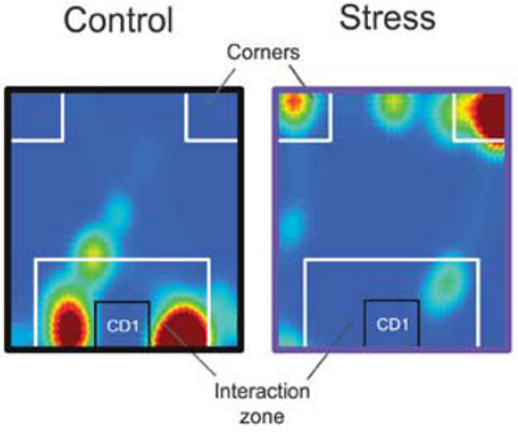

c

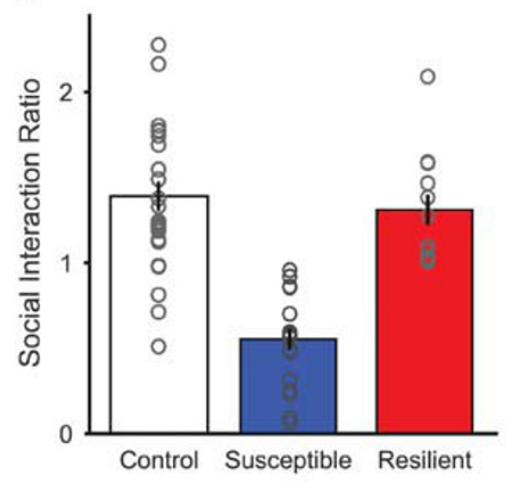

d

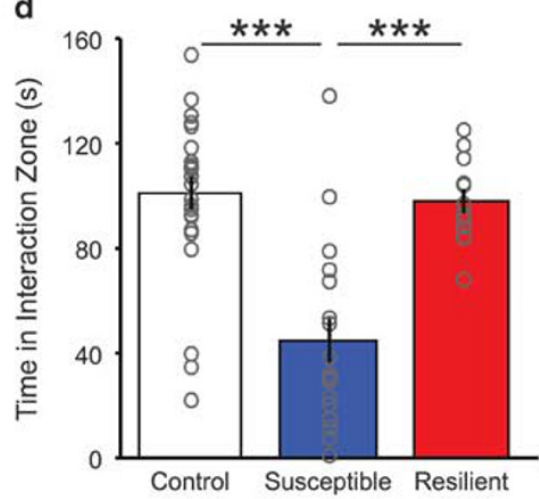

e

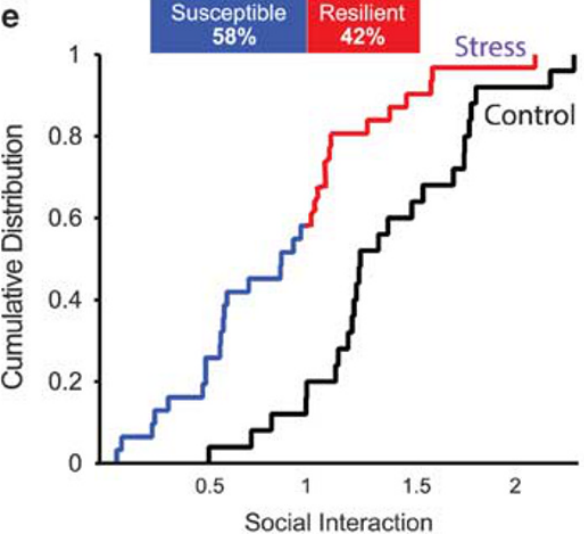

Figure 2 Repeated social defeat results in social avoidance. (a) Time spent interacting with an empty cup (non-social target) and a novel mouse (social target) after chronic social defeat stress (Kruskal-Wallis, $\chi^{2}=15.3 ; P=0.0015$, Wilcoxon-rank sum, $* * * *<<0.00 I$; control $n=25$, stressed $n=30$ ). (b) Right, social interaction ratios for control and stressed mice (Wilcoxon-rank sum, $* * * P<0.00$ I; control $n=25$, stressed $n=30$ ). Left, heat maps indicating time spent in the interaction chamber for example control and stress mice. (c) Social interaction ratios for control $(n=25)$, susceptible $(n=17$, social interaction ratios $<1)$, and resilient $(n=13$, social interaction ratios $>1)$ mice. (d) Time spent in the interaction zone with social target for control $(n=25)$, susceptible $(n=17)$, and resilient $(n=13)$ mice (Kruskal-Wallis $\chi^{2}=20.7 ; P<0.00003$, Wilcoxon-rank sum, **** $P<0.00 \mathrm{I}$ ). (e) Top, the percent of resilient and susceptible mice. Bottom, the cumulative distribution of SI values for control (black line) and stressed mice. Blue line indicates susceptible and red line indicates resilient mice. Error bars represent SEM.

Materials and Methods; Figure 2b; Wilcoxon-rank sum $P<0.0001)$. We conducted our experiments in 3 different female cohorts. In all of the cohorts, the socially defeated female mice had decreased SI ratios relative to unstressed controls (cohort 1: SI ratios 1.2 vs 0.8 , Wilcoxon-rank sum, $P=0.012, n=10,5$; cohort 2 : SI ratios 1.6 vs 1.2 , Wilcoxonrank sum $P=0.054, n=10,8$; cohort 3 : SI ratios 1.3 vs 0.8 , rank sum $P=0.050, n=5,17)$. These data suggest that our findings are highly replicable. Mice who prefer interacting with a social target over an inanimate object have a SI ratio $>1$, whereas conversely those that prefer the inanimate object have a SI ratio $<1$. As a result, prior studies have identified mice with a SI ratio of $<1$ as susceptible and those with an SI ratio of $>1$ as resilient (Golden et al, 2011; Krishnan et al, 2007). Splitting the data in this way has revealed substantial molecular, cellular and electrophysiological differences between susceptible and resilient mice (Chaudhury et al, 2013; Friedman et al, 2014; Krishnan et al, 2007; Lobo et al, 2013). Using these definitions, female mice exposed to CSDS also formed susceptible and resilient groups, with susceptible mice spending significantly less time exploring a novel social target than resilient mice (Figure 2d; Kruskal-Wallis, $\chi^{2}=20.7 ; P<0.00003$ for main effect of group, post hoc Wilcoxon-rank sum $P<0.00001$ control $v s$ susceptible $(n=25,17), P<0.0001$ susceptible $v s$ resilient $(n=17,13), P=0.28$ control vs resilient $(n=25,13)$, Bonferroni corrected critical value $=0.0167$ ). Corticosterone levels of stressed mice on the first day of social defeat inversely correlated with the social interaction outcome (Pearson's $r=-0.39, P=0.047$ ), suggesting that lower SI ratios were associated with higher reactivity to the social defeat stress. As can be seen from the cumulative distribution of the SI values of control and stressed mice, $58 \%$ of the female mice are susceptible (Figure 2e). Previous studies of socially defeated males have produced comparable percentages of susceptible mice ( $55 \%$ ) (Challis et al, 2013; Francis et al, 2015; Krishnan et al, 2007).

In male mice, CSDS reduces exploration of a novel mouse both immediately (1 day after CSDS) and persistently (4 weeks after CSDS) (Krishnan et al, 2007). We tested whether CSDS has a long-lasting impact on female mice by re-testing social interaction 40 days after the conclusion of CSDS in a subset of mice. We found that CSDS also reduces the social interaction ratio at this later time point $(n=3,14$ Wilcoxon-rank sum $P=0.047$, Supplementary Figure 3). 
To determine the impact of CSDS on the pursuit of rewarding stimuli in female mice, we tested sucrose preference. As has been previously reported for male mice (Chaudhury et al, 2013; Krishnan et al, 2007), susceptible female mice exhibited decreased sucrose preference, whereas resilient mice preferred sucrose at control levels (Figure 3a; Kruskal-Wallis, $\chi^{2}=8.6 ; P<0.01$ for main effect of group, post hoc Wilcoxon-rank sum $P<0.01$ control vs susceptible $(n=24,15), P<0.015$ for susceptible $v s$ resilient $(n=15,10)$, $P>0.98$ for control $v s$ resilient $(n=24,10)$, Bonferronicorrected critical value $=0.0167)$. Sucrose preference positively correlated with SI score in stressed mice $(r=0.4$, $P<0.5)$, but not in control mice $(r=0.1, \quad P>0.6$; Supplementary Figure 4).

To assess anxiety-like behavior, we measured the time mice spent exploring the open arms of an EPM. Chronically defeated mice explore the open arms less than control mice (Figure 3b; Wilcoxon-rank sum $P=0.035(n=14,21)$ ). Interestingly, there was no significant difference in open arm exploration between susceptible and resilient mice (Figure 3c; Wilcoxon-rank sum $P=0.47 \quad(n=13,8)$, Supplementary Figure 5), suggesting that the anhedonic and anxiogenic effects of CSDS are separable.

This paradigm yields susceptible and resilient cohorts of female mice. However, it is important to rule out other potential sources of experimental variability that could lead to apparent susceptible and resilient outcomes. First, both susceptible and resilient cohorts experience comparable days of aggression (Figure 4; Kolmogorov-Smirnov test, $P=0.63$ ). Second, although VTA dopaminergic activity reportedly influences SI (Gunaydin et al, 2014) and varies with estrous cycle phase (Calipari et al, 2017), estrous cycle phase did not impact social exploration in either unstressed mice (Figure 4b, left; Wilcoxon-rank sum $P=0.4(n=11,6)$ ) or in chronic social defeated mice (Figure $4 \mathrm{~b}$, right; Wilcoxonrank sum $P=0.72 \quad(n=8,6)$. Finally, to ensure that behavioral effects in females result from repeated aggression rather than the application of male scent, we compared the effects of male scent application on behavior in control mice. We found no effect of the application of male scent alone on corticosterone levels, social exploration, and sucrose preference (Supplementary Figure 6).

\section{DISCUSSION}

There is increasing recognition of the need to include female subjects in both pre-clinical research and clinical trials (Miller et al, 2017). CSDS has proven a powerful tool for dissecting the mechanisms underlying stress susceptibility
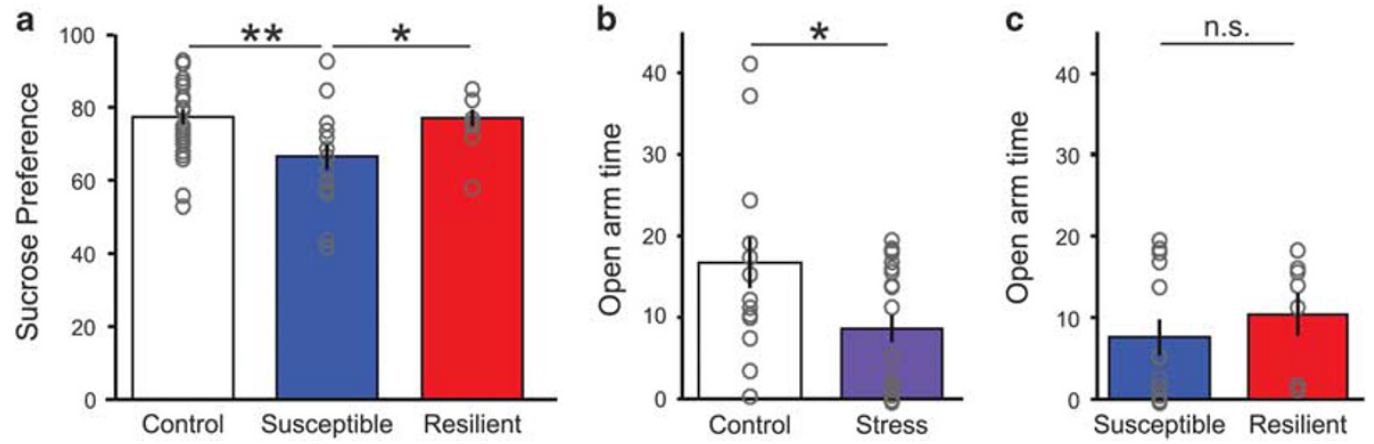

Figure 3 Repeated social defeat results in decreased sucrose preference and open arm exploration. (a) Sucrose preference for control, susceptible and resilient mice (Kruskal-Wallis, $\chi^{2}=8.6$; Wilcoxon-rank sum, $* * P<0.01 ; n=24,10,16$ ). (b) Time spent by control and stressed mice in the open arms of the elevated plus maze (Wilcoxon-rank sum, *P $<0.05 ; n=\mid 4,21$ ). (c) Time spent in the EPM by susceptible and resilient mice (not significant (n.s.); $n=8$, I4). Error bars represent SEM.
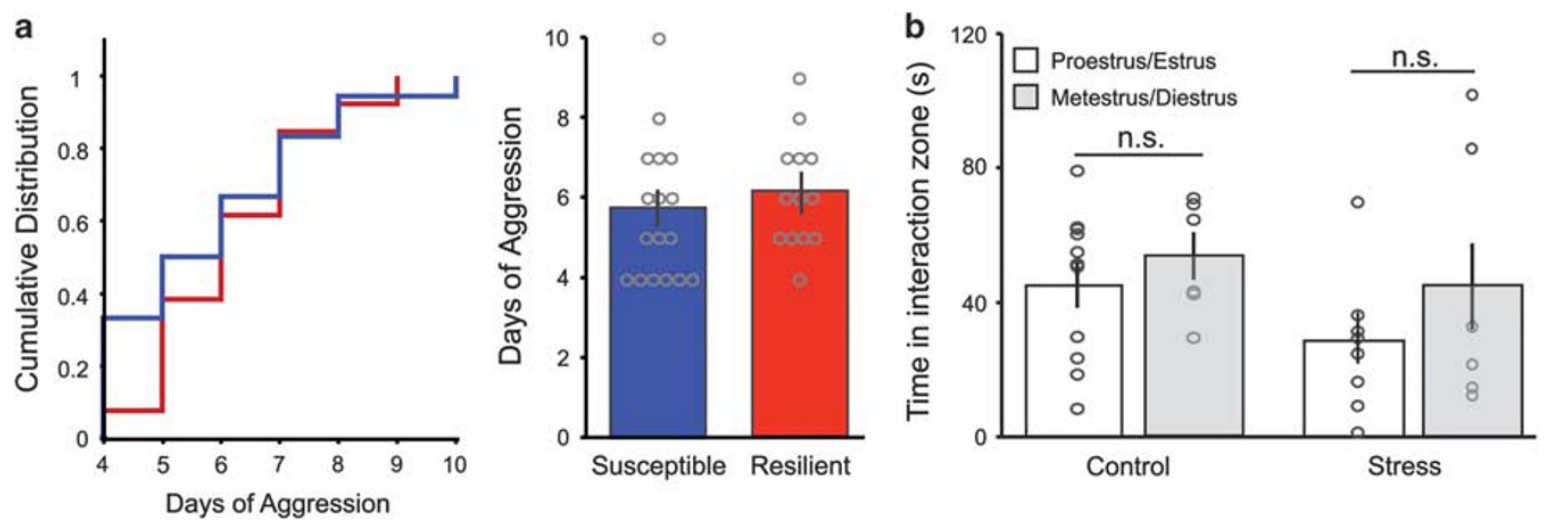

Figure 4 Neither number of aggressive encounters nor estrous cycle determines susceptible outcome. (a) Left, cumulative distribution of days of aggression for susceptible and resilient mice (Kolmogorov-Smimov test, $P=0.63$ ). Right, distribution of aggressive days grouped by susceptible vs resilient $(n=13-18)$. (b) Social interaction times for control and chronically stressed mice across estrous phase (not significant (n.s.); $n=6-1 \mathrm{I}$ ). Error bars represent SEM. 
and resilience (Chaudhury et al, 2013; Francis et al, 2015; Friedman et al, 2014; Krishnan et al, 2007). However, the inability to include females in these studies has posed a serious limitation. In this paper, we present an easy-to-use method for conducting CSDS in female mice. We demonstrate that this paradigm impacts social exploration, sucrose preference and anxiety-like behavior in female mice. Moreover, it yields groups of susceptible and resilient mice that differ in social interaction and sucrose preference but exhibit similar anxiety-like responses. We also find that estrous cycle phase does not significantly impact the ability to elicit male aggression and does not modulate social exploration, the primary assay of CSDS. These results should mitigate concerns about behavioral variability in female mice due to hormonal changes. Interestingly, although other chronic stress paradigms cause females to stop cycling (Breen et al, 2012), CSDS did not disrupt estrous cycle (data not shown). The absence of a stress-induced effect on estrous cycle is likely due to the continuous presence of male mice and urine throughout the stress paradigm since both male mice and the proteins found in their urine suffice to induce estrus in female mice (Jemiolo et al, 1986; Whitten, 1966; Whitten et al, 1968). Overall, our findings strikingly resemble those seen in chronic socially defeated male mice (Krishnan et al, 2007) and provide the foundation for parallel studies comparing the underpinnings of susceptibility and resilience in males and females.

In this study, we used male urine to induce aggressive interactions between males and females. Mugford and Nowell, 1970 demonstrated that applying urine from aggressive males to the backs of castrated males increased resident-intruder aggression, whereas applying female urine decreased the number of attacks (Mugford and Nowell, 1970). In a different study, male urine had a pro-aggressive effect on female behavior (Palanza et al, 1994). Further work has identified the responsible odorants as two major urinary proteins and highlighted the role of the vomeronasal circuit (Chamero et al, 2007) and ventral medial hypothalamus (VMH) for generating this aggressive behavior (Falkner et al, 2014; Lin et al, 2011; Maruniak et al, 1986).

Although female mouse aggression is rarely observed under standard laboratory conditions, ethologic studies reveal that female aggressive interactions do occur under a variety of circumstances. Female mice attack both males and females to maintain population size (Chovnick et al, 1987; Yasukawa et al, 1985) and to defend their nests in the postpartum period (Haney et al, 1989). Moreover, male mice that establish territory together with a pregnant or lactating female attack both male and female intruders (Palanza et al, 1996). Indeed, females across many species in addition to mice, including rats (DeBold and Miczek, 1984), blackbirds ((Yasukawa and Searcy, 1982), and non-human primates (Huchard and Cowlishaw, 2011; Isbell, 1991) engage in aggressive interactions. Moreover, acute and chronic stress can increase female aggression in rats (Albonetti and Farabollini, 1993; Cordero et al, 2013). Thus, female aggressive experience represents an ethologically sound stressor.

Nonetheless, because standard laboratory conditions rarely reveal aggressive behavior towards females, few studies have examined CSDS in female rodents (Solomon, 2017) and no published studies exist for conducting CSDS in females in the commonly used and genetically tractable Mus musculus. Existing methods for conducting social defeat stress in females include using monogamous species such as the California mouse, whose females show territorial aggression. Importantly, unlike Mus musculus mice, male California mice do not develop social deficits in response to chronic defeat, making it difficult to directly compare the underpinnings of stress susceptibility in males and females.

Lactating rat (Haller, 1999) and mouse (Jacobson-Pick et al, 2013) dams attack intruders to their nests. This approach has been successfully exploited to chronically defeat female rats (Haller, 1999) and to acutely defeat female mice (Jacobson-Pick et al, 2013). However, implementing this method on a large scale poses substantial logistical challenges, as it involves maintaining large colonies of similarly timed pregnancies to generate sufficient lactating aggressors.

A recent study by Russo et al (2016) cleverly exploited the recently discovered $\mathrm{VMH}$ neurons that promote aggression (Lee et al, 2014; Lin et al, 2011) to generate aggressors for conducting CSDS. This method also induces intermittent aggression against females and consequent social avoidance. However, this approach requires stereotactic targeting of the $\mathrm{VMH}$ with a virus and daily drug administration to activate aggression. We believe our paradigm is more accessible as it relies on readily available mice and requires no surgery or reagents.

Despite its advantages, our technique has limitations. First, although female aggression is part of the natural behavioral repertoire of mice (Chovnick et al, 1987; Haney et al, 1989; Palanza et al, 1996; Yasukawa et al, 1985), our method induces this experience in an artificial manner. Second, female mice exhibit sex-specific stress responses, such as disruption of the estrous cycle (Breen et al, 2012), yet in our approach these effects are likely masked by the continuous presence of males (Jemiolo et al, 1986; Whitten et al, 1968). Third, although we have modeled it on a male protocol, it differs in a few respects: the number of aggressive bouts per session ( 2 in females; 5-10 in males (Golden et al, 2011)) and the number of days of aggression (average 5 vs 10). These differences limit the ability to compare the impact of the exact same stressors in males and females. That both male and female paradigms produce comparable behavioral outcomes mitigates this concern. Fourth, our method produces varying levels of aggression, ranging from 1 to 10 days. Although we show that susceptibility does not depend on the supra-threshold days of aggression (Supplementary Figure 2; Figure 4), this protocol results in $20 \%$ of mice that undergo sub-threshold stress. Subthreshold social defeat stress has been used as an experimental manipulation for testing circuit interventions on susceptibility (Chaudhury et al, 2013; Francis et al, 2015). These mice could thus potentially be used as a separate experimental group. Finally, the need to collect urine and apply it to female mice makes this method slightly more time consuming than CSDS in males.

Are female mice more susceptible than males? At first glance, male and female mice show comparable rates of susceptibility to CSDS (58 vs 55\%). It is worth noting that females develop susceptibility after 4 days, whereas a recent study reported that male mice do not develop social deficits with up to 5 days of social defeat (Wook Koo et al, 2016). 
The concept that female susceptibility manifests in response to fewer days of stress is consistent with other work comparing male and female responses to chronic stress. For instance, one study showed that female mice succumbed to chronic variable stress after 7 days, whereas males required 21 days (Hodes et al, 2015).

In future experiments, we plan to test whether we can improve the efficiency of the paradigm by pooling urine from multiple aggressive mice, using the identified major urinary proteins that elicit aggression to increase the aggression toward female subjects (Chamero et al, 2007), using new resident aggressors for each cohort of females, or selecting only those male mice that consistently demonstrate aggressive behavior towards females. More importantly, we will begin testing the molecular and physiological underpinnings of stress susceptibility and resilience in female mice. Exciting recent molecular work has identified unique genes that are dysregulated in stressed male and female mice (Hodes et al, 2015). Our method makes it possible to test the contributions of these genes and other identified cellular and circuit mechanisms that mediate female susceptibility and resilience.

\section{FUNDING AND DISCLOSURE}

This work was supported by grants from the NIMH to AZH (K08 MH109735), and EDL (R01 MH081968); by the Hope for Depression Research Foundation (JAG, AZH). This article was prepared while Joshua A. Gordon was employed at Columbia University and the New York State Psychiatric Institute. The authors declare no conflict of interest.

\section{ACKNOWLEDGMENTS}

We would like to thank members of the Gordon and Hen labs for technical assistance and discussions. AZH designed and analyzed the experiments. PA, ZHB, and AZH performed the experiments and analyzed the data. ESH, RA, MPM, AIA, SSB, EDL, RH, and JAG assisted with the design, implementation, analysis, and interpretation of experiments. AZH, PA, and JAG interpreted the results and wrote the paper.

\section{DISCLAIMER}

The opinions expressed in this article are the author's own and do not reflect the view of the National Institutes of Health, the Department of Health and Human Services, or the United States government.

\section{REFERENCES}

Albonetti ME, Farabollini F (1993). Restraint increases both aggression and defence in female rats tested against same-sex unfamiliar conspecifics. Aggressive Behav 19: 369-376.

Autry AE, Adachi M, Cheng P, Monteggia LM (2009). Genderspecific impact of brain-derived neurotrophic factor signaling on stress-induced depression-like behavior. Biol Psychiatry 66: 84-90.

Beery AK, Zucker I (2011). Sex bias in neuroscience and biomedical research. Neurosci Biobehav Rev 35: 565-572.
Berton O, McClung CA, Dileone RJ, Krishnan V, Renthal W, Russo SJ et al (2006). Essential role of BDNF in the mesolimbic dopamine pathway in social defeat stress. Science 311: 864-868.

Bourke CH, Neigh GN (2012). Exposure to repeated maternal aggression induces depressive-like behavior and increases startle in adult female rats. Behav Brain Res 227: 270-275.

Breen KM, Thackray VG, Hsu T, Mak-McCully RA, Coss D, Mellon PL (2012). Stress levels of glucocorticoids inhibit LHbeta-subunit gene expression in gonadotrope cells. Mol Endocrinol 26: $1716-1731$.

Byers SL, Wiles MV, Dunn SL, Taft RA (2012). Mouse estrous cycle identification tool and images. PLOS ONE 7: e35538.

Caligioni CS (2009). Assessing reproductive status/stages in mice. Curr Protoc Neurosci Suppl 48: A.4I.1-A.4I.8.

Calipari ES, Juarez B, Morel C, Walker DM, Cahill ME, Ribeiro E et al (2017). Dopaminergic dynamics underlying sex-specific cocaine reward. Nat Commun 8: 13877.

Challis C, Boulden J, Veerakumar A, Espallergues J, Vassoler FM, Pierce RC et al (2013). Raphe GABAergic neurons mediate the acquisition of avoidance after social defeat. J Neurosci 33: 13978-13988.

Chamero P, Marton TF, Logan DW, Flanagan K, Cruz JR, Saghatelian A et al (2007). Identification of protein pheromones that promote aggressive behaviour. Nature 450: 899-902.

Chaudhury D, Walsh JJ, Friedman AK, Juarez B, Ku SM, Koo JW et al (2013). Rapid regulation of depression-related behaviours by control of midbrain dopamine neurons. Nature 493: 532-536.

Chovnick A, Yasukawa NJ, Monder H, Christian JJ (1987). Female behavior in populations of mice in the presence and absence of male hierarchy. Aggressive Behav 13: 367-375.

Cordero MI, Ansermet F, Sandi C (2013). Long-term programming of enhanced aggression by peripuberty stress in female rats. Psychoneuroendocrinology 38: 2758-2769.

DeBold JF, Miczek KA (1984). Aggression persists after ovariectomy in female rats. Horm Behav 18: 177-190.

Falkner AL, Dollar P, Perona P, Anderson DJ, Lin D (2014). Decoding ventromedial hypothalamic neural activity during male mouse aggression. J Neurosci 34: 5971-5984.

Francis TC, Chandra R, Friend DM, Finkel E, Dayrit G, Miranda J et al (2015). Nucleus accumbens medium spiny neuron subtypes mediate depression-related outcomes to social defeat stress. Biol Psychiatry 77: 212-222.

Friedman AK, Walsh JJ, Juarez B, Ku SM, Chaudhury D, Wang J et al (2014). Enhancing depression mechanisms in midbrain dopamine neurons achieves homeostatic resilience. Science 344: 313-319.

Golden SA, Covington HE 3rd, Berton O, Russo SJ (2011). A standardized protocol for repeated social defeat stress in mice. Nat Protoc 6: 1183-1191.

Gruene TM, Flick K, Stefano A, Shea SD, Shansky RM (2015). Sexually divergent expression of active and passive conditioned fear responses in rats. Elife 4: e11352.

Gunaydin LA, Grosenick L, Finkelstein JC, Kauvar IV, Fenno LE, Adhikari A et al (2014). Natural neural projection dynamics underlying social behavior. Cell 157: 1535-1551.

Haller J (1999). Defeat is a major stressor in males while social instability is stressful mainly in females: towards the development of a social stress model in female rats. Brain Res Bull 50: 33-39.

Haney M, Debold JF, Miczek KA (1989). Maternal aggression in mice and rats towards male and female conspecifics. Aggressive Behav 15: 443-453.

Hodes GE, Pfau ML, Purushothaman I, Ahn HF, Golden SA, Christoffel DJ et al (2015). Sex differences in nucleus accumbens transcriptome profiles associated with susceptibility versus resilience to subchronic variable stress. J Neurosci 35: 16362-16376.

Huchard E, Cowlishaw G (2011). Female-female aggression around mating: an extra cost of sociality in a multimale primate society. Behav Ecol 22: 1003-1011. 
Isbell LA (1991). Contest and scramble competition: patterns of female aggression and ranging behavior among primates. Behav Ecol 2: 143-155.

Jacobson-Pick S, Audet MC, McQuaid RJ, Kalvapalle R, Anisman H (2013). Social agonistic distress in male and female mice: changes of behavior and brain monoamine functioning in relation to acute and chronic challenges. PLoS ONE 8: e60133.

Jemiolo B, Harvey S, Novotny M (1986). Promotion of the Whitten effect in female mice by synthetic analogs of male urinary constituents. Proc Natl Acad Sci USA 83: 4576-4579.

Kessler RC, Berglund P, Demler O, Jin R, Koretz D, Merikangas KR et al (2003). The epidemiology of major depressive disorder: results from the National Comorbidity Survey Replication (NCS-R). JAMA 289: 3095-3105.

Kessler RC, McGonagle KA, Zhao S, Nelson CB, Hughes M, Eshleman $S$ et al (1994). Lifetime and 12-month prevalence of DSM-III-R psychiatric disorders in the United States: results from the National Comorbidity Survey. Arch Gen Psychiatry 51: 8-19.

Kokras N, Dalla C (2014). Sex differences in animal models of psychiatric disorders. Br J Pharmacol 171: 4595-4619.

Krishnan V, Han MH, Graham DL, Berton O, Renthal W, Russo SJ et al (2007). Molecular adaptations underlying susceptibility and resistance to social defeat in brain reward regions. Cell 131: 391-404.

Kudryavtseva NN, Bakshtanovskaya IV, Koryakina LA (1991). Social model of depression in mice of C57BL/6J strain. Pharmacol Biochem Behav 38: 315-320.

Larrieu T, Cherix A, Duque A, Rodrigues J, Lei H, Gruetter R et al (2017). Hierarchical status predicts behavioral vulnerability and nucleus accumbens metabolic profile following chronic social defeat stress. Curr Biol 27: 2202-2210.

Lebron-Milad K, Milad MR (2012). Sex differences, gonadal hormones and the fear extinction network: implications for anxiety disorders. Biol Mood Anxiety Disord 2: 3.

Lee H, Kim DW, Remedios R, Anthony TE, Chang A, Madisen L et al (2014). Scalable control of mounting and attack by Esr1+ neurons in the ventromedial hypothalamus. Nature 509: 627-632.

Lin D, Boyle MP, Dollar P, Lee H, Lein ES, Perona P et al (2011). Functional identification of an aggression locus in the mouse hypothalamus. Nature 470: 221-226.

Lobo MK, Zaman S, Damez-Werno DM, Koo JW, Bagot RC, DiNieri JA et al (2013). DeltaFosB induction in striatal medium spiny neuron subtypes in response to chronic pharmacological, emotional, and optogenetic stimuli. J Neurosci 33: 18381-18395.

Maruniak J, Wysocki C, Taylor J (1986). Mediation of male mouse urine marking and aggression by the vomeronasal organ. Physiol Behav 37: 655-657.

McGill TE (1962). Sexual behavior in three inbred strains of mice. Behaviour 19: 341-350.

Miczek KA, O'Donnell JM (1978). Intruder-evoked aggression in isolated and nonisolated mice: effects of psychomotor stimulants and L-dopa. Psychopharmacology 57: 47-55.
Miller LR, Marks C, Becker JB, Hurn PD, Chen WJ, Woodruff T et al (2017). Considering sex as a biological variable in preclinical research. FASEB J 31: 29-34.

Mugford R, Nowell N (1970). Pheromones and their effect on aggression in mice. Nature 226: 967-968.

Palanza P, Parmigiani S, vom Saal FS (1994). Male urinary cues stimulate intra-sexual aggression and urine-marking in wild female mice, Mus musculus domesticus. Anim Behav 48: 245-247.

Palanza P, Re L, Mainardi D, Brain P, Parmigiani S (1996). Male and female competitive strategies of wild house mice pairs (Mus musculus domesticus) confronted with intruders of different sex and age in artificial territories. Behaviour 133: 863-882.

Perez-Escudero A, Vicente-Page J, Hinz RC, Arganda S, de Polavieja GG (2014). idTracker: tracking individuals in a group by automatic identification of unmarked animals. Nat Methods 11: 743-748.

Russo SJ, Nestler EJ (2013). The brain reward circuitry in mood disorders. Nat Rev Neurosci 14: 609-625.

Russo SJ, Takahashi A, Zhang H, Zhang S, Grossman Y, Aleyasin H, Flanigan ME, Pena C, Pfau ML, Hodes GE, Menard C, Nestler EJ, Han MH(eds) (2016). Establishment of repeated social defeat stress model in female mice. 2016 San Diego: San Diego.

Shansky RM (2015). Sex differences in PTSD resilience and susceptibility: challenges for animal models of fear learning. Neurobiol Stress 1: 60-65.

Shansky RM, Hamo C, Hof PR, Lou W, McEwen BS, Morrison JH (2010). Estrogen promotes stress sensitivity in a prefrontal cortex-amygdala pathway. Cereb Cortex 20: 2560-2567.

Solomon MB (2017). Evaluating social defeat as a model for psychopathology in adult female rodents. J Neurosci Res 95: 763-776.

Trainor BC (2011). Stress responses and the mesolimbic dopamine system: social contexts and sex differences. Horm Behav 60: 457-469.

Trainor BC, Pride MC, Villalon Landeros R, Knoblauch NW, Takahashi EY, Silva AL et al (2011). Sex differences in social interaction behavior following social defeat stress in the monogamous California mouse (Peromyscus californicus). PLoS ONE 6: e17405.

Whitten W (1966). Pheromones and mammalian reproduction. Adv Reprod Physiol 1: 155-15.

Whitten WK, Bronson FH, Greenstein JA (1968). Estrus-inducing pheromone of male mice: transport by movement of air. Science 161: $584-585$.

Wook Koo J, Labonte B, Engmann O, Calipari ES, Juarez B, Lorsch $\mathrm{Z}$ et al (2016). Essential role of mesolimbic brain-derived neurotrophic factor in chronic social stress-induced depressive behaviors. Biol Psychiatry 80: 469-478.

Yasukawa K, Searcy WA (1982). Aggression in female Red-Winged Blackbirds: a strategy to ensure male parental investment. Behav Ecol Sociobiol 11: 13-17.

Yasukawa NJ, Monder H, Leff FR, Christian JJ (1985). Role of female behavior in controlling population growth in mice. Aggressive Behav 11: 49-64.

Supplementary Information accompanies the paper on the Neuropsychopharmacology website (http://www.nature.com/npp) 